\title{
Fibra de cultivares de algodoeiro sob condições de sequeiro
}

\section{Fiber of cotton plant cultivar under drought conditions}

\author{
Antônia Samia Lima ${ }^{1}$, Maria Eugenia da Costa², José Jaime Vasconcelos Cavalcanti ${ }^{3}$, Ana Paula \\ Medeiros dos Santos Rodrigues ${ }^{4}$, Antonio Francisco de Mendonça Júnior ${ }^{5}$
}

${ }^{1}$ Faculdade de Tecnologia do Instituto Centro de Ensino Tecnológico, Sertão Central, aslima@gmail.com; ${ }^{2}$ Faculdade de Tecnologia do Instituto Centro de Ensino Tecnológico, Sertão Central, eugenia.ufersa@gmail.com; ${ }^{3}$ EMBRAPA Algodão, Campina Grande, Paraíba, jaime.cavalcanti@embrapa.br;

${ }^{4}$ Universidade Estadual do Rio Grande do Norte, Mossoró, Rio Grande do Norte, anapaulamsr@ yahoo.com.br; ${ }^{5}$ Universidade Federal Rural de Pernambuco, Recife, Pernambuco, agromendoncajr@yahoo.com.br

\section{N O T A}

Recebido: $10 / 11 / 2018$

Aprovado: 21/12/2018

\section{Palavras-chave:}

Algodão

Produção de pluma

Variabilidade fenotípica

Key words:

Cotton

Phenotypic variability

Plume production

\begin{abstract}
R E S U M O
Objetivando avalizar as características de fibra do diferentes cultivares de algodão realizouse uma pesquisa Campo Experimental da Fazenda Escola da Faculdade de Tecnologia do Instituto Centro de Ensino Tecnológico, Sertão Central, no município de Quixeramobim, Ceará, cujas coordenadas geográficas Latitude $(\mathrm{S}) 05^{\circ} 11^{\prime} 57^{\prime}$ 'S, Longitude $(\mathrm{W}) 39^{\circ} 17^{\prime} 34^{\prime \prime} \mathrm{W}$ e Altitude (M) 191. O regime de chuvas local varia de 700 a $750 \mathrm{~mm}$. Estas chuvas se concentram no período de janeiro a junho. A época do plantio de Quixeramobim, foi em fevereiro caracterizando como safra normal. O delineamento experimental utilizado para o ensaio foi o de blocos ao acaso com quatro repetições e vinte tratamentos, sendo que a parcela experimental foi constituída por quatro linhas de 5 metros linear, com espaçamento entre linhas de 0,90 metros, estande entre 6 a 9 plantas $/ \mathrm{m}$. A área útil da parcela utilizada para obtenção dos dados, foram as duas linhas centrais, nesse ensaio utilizou-se as linhagens e ou cultivares referente ao ano/safra 2019. Concluiu-se que há variabilidade fenotípica os tratamentos apresentaram diferenças significativas para a maioria dos caracteres e as cultivares BRS 416, BRS 286 e BRS 293 são as de melhores desempenhos pois associaram as melhores médias para produtividade de pluma e para as características de fibras.
\end{abstract}

\section{A B S T R A C T}

In order to evaluate the fiber characteristics of the different cotton cultivars, a research field was carried out in the Quixeramobim, Ceará, municipality, where the geographical coordinates Latitude (S ) $05^{\circ} 11^{\prime} 57$ "S, Longitude (W) $39^{\circ}$ 17'34"W and Altitude (M) 191. The local rainfall varies from 700 to $750 \mathrm{~mm}$. These rains are concentrated in the period from January to June. The time of the Quixeramobim planting, was in February characterizing like normal crop. The experimental design was a randomized complete block design with four replications and twenty treatments. The experimental plot consisted of four linear 5-meter lines, spacing 0.90 meters between rows, 6 to 9 plants / $\mathrm{m}$. The useful area of the plot used to obtain the data were the two central lines, in this test the strains and or cultivars were used referring to the year/crop 2019. It was concluded that there are phenotypic variability the treatments presented significant differences for the majority BRS 416, BRS 286 and BRS 293 are the best performance because they associated the best averages for feather productivity and fiber characteristics.

\section{INTRODUÇÃO}

O algodoeiro herbáceo (Gossypium hirsutum L.) é a principal fonte comercial de fibra natural para a indústria têxtil em todo o mundo. A lavoura é cultivada em mais de 60 países, em área superior a 35 milhões de hectares, com uma produção de 24 milhões de toneladas de fibra, destacando-se Índia, China, Estados Unidos, Paquistão e Brasil como maiores produtores mundiais (USDA, 2018). O agronegócio do algodoeiro envolve mais de 350 milhões de pessoas em sua cadeia produtiva, gerando renda anual de aproximadamente US\$ 12 bilhões (ABRAPA, 2018).
No ano de 2017, foi plantado no Brasil 939,1 mil hectares de algodão, gerando uma produção de 1.529,5 mil toneladas de fibra. A região dos cerrados do Centro-Oeste e Nordeste é a principal produtora de algodão, detendo mais de $97,6 \%$ da produção nacional (CONAB, 2018). Essa região é caracterizada pela topografia plana que permite a mecanização completa das atividades, com alto nível tecnológico e estações chuvosa e seca bem definidas, proporcionando produção e qualidade da fibra em níveis competitivos para atender as demandas das indústrias têxteis nacionais. Apesar dessas condições, as elevadas temperaturas

\section{Revista Verde}

ISSN 1981-8203

Pombal, Paraíba, Brasil v. 14, n.1, jan.-mar, p.166-169, 2019 doi: 10.18378/rvads.v14i1.6718 
com curtos períodos de estiagens afetam a produtividade da cultura, independente da região em que é cultivada (PETTIGREW, 2004; AQUINO et al., 2012; ZONTA et al., 2015). O melhoramento genético tem oferecido grande contribuição para minimizar problemas oriundos de estresses abióticos, desenvolvendo cultivares de larga adaptação ambiental e estabilidade produtiva (FREIRE et al., 2008; CARVALHO et al., 2011; CARVALHO et al., 2015).

De acordo com Ullah et al. (2017), a falta de água pode afetar o crescimento, a produtividade e a qualidade da fibra de algodão, contudo, ao longo de sua evolução, o algodoeiro desenvolveu diferentes mecanismos para aumentar a tolerância à seca, tais como regulação estomática e fotossintética, aumento do sistema radicular, ajustamento osmótico e outros mecanismos bioquímicos e moleculares. Os autores afirmam que, dentre outras estratégias, o melhoramento genético tradicional e o desenvolvimento de variedades transgênicas são de fundamental importância para aumentar a tolerância do algodão ao estresse hídrico.

Considerando que a seca é um fenômeno que ocorre em nível mundial, o investimento de pesquisa no desenvolvimento de cultivares tolerantes a ambientes com restrição hídrica torna-se imperioso, especialmente vislumbrando a tendência crescente das mudanças climáticas que tem ocorrido nos diferentes hemisférios.

Historicamente a cultura do algodão na região Nordeste atingiu o seu ápice de importância econômica e social na década de setenta quando foram cultivados 3.247 mil hectares, sendo 2.562,19 mil hectares de algodoeiros arbóreos e 684,91 mil hectares de algodoeiro herbáceo. Naquela época a cultura era responsável pela geração de mais de um milhão de empregos no campo e fornecia matéria prima para 259 algodoeiras (FREIRE et al., 2008).

$\mathrm{O}$ ensaio de cultivares de algodão em diferentes densidades no semiárido em condições de sequeiro de Algodoeiro Herbáceo conduzido pelo programa de Melhoramento da Embrapa Algodão na Região Nordeste é extremamente importante para a tomada de decisão no processo de seleção e recomendação de novas cultivares, tendo em vista a elevada exigência dos produtores por cultivares de alta performance agronômica e tecnológica da fibra, e que atenda ao novo modelo de sustentabilidade ambiental, visando aumentar a eficiência no processo de escolha de uma nova cultivar.

O objetivo desta pesquisa foi avaliar o comportamento produtivo e a qualidade de fibra de diferentes cultivares de algodoeiro, em condições de sequeiro Semiárido Cearense.

\section{MATERIAL E MÉTODOS}

A pesquisa foi conduzida do Campo Experimental da Fazenda Escola da Faculdade de Tecnologia CENTEC/FATEC, Sertão Central (FATEC-SC), no município de Quixeramobim-CE, cujas coordenadas geográficas Latitude (S) $05^{\circ} 11^{\prime} 57^{\prime}$ 'S, Longitude (W) $39^{\circ} 17^{\prime} 34^{\prime \prime}$ W e Altitude (M) 191. O regime de chuvas local varia de 700 a $750 \mathrm{~mm}$. Estas chuvas se concentram no período de janeiro a junho. A época do plantio de Quixeramobim, foi em fevereiro caracterizando como safra normal.

Para a pesquisa foram utilizado os dados provenientes do ensaio de cultivares de algodão em diferentes densidades no semiárido em condições de sequeiro oriundos do
Programa de Melhoramento para as condições de sequeiro da Embrapa Algodão, conduzidos no ano agrícola 2018, no município de Quixeramobim. (Tabela1). Sendo usado os seguintes materiais genéticos: BRS 293, BRS 286, BRS 416, BRS 371 RF e BRS Aroeira, todas originadas do programa de melhoramento genético da EMBRAPA.

$\mathrm{O}$ delineamento experimental utilizado para o ensaio foi o de blocos ao acaso com quatro repetições e vinte tratamentos, sendo que a parcela experimental foi constituída por quatro linhas de 5 metros linear, com espaçamento entre linhas de 0,90 metros, estande entre 6 a 9 plantas $/ \mathrm{m}$. A área útil da parcela utilizada para obtenção dos dados, foram as duas linhas centrais, nesse ensaio utilizou-se as linhagens e ou cultivares referente ao ano/safra 2019. Na colheita manual fez-se a amostragem de 20 capulhos/parcela, que foram ensacados e identificados com a numeração de cada parcela, encaminhados para pesagem e beneficiamento em um descaroçador de algodão de pequenas amostras e, em seguida, obteve-se o rendimento de fibra de cada amostra através da divisão do peso da fibra pelo peso total da amostra e multiplicado por $100(\mathrm{~F} / \mathrm{T}) \times 100$ onde, F correspondeu ao peso da Fibra e T, o peso total.

As características tecnológicas de fibras de cada amostra da parcela foram analisadas através do equipamento HVI (High Volume Instruments), do Laboratório de Tecnologia de Fibras da Embrapa Algodão, localizada em Capina Grande, Paraíba. O HVI é padronizado mundialmente para análise e classificação de algodão, conforme a Instrução Normativa 63/2002, do Ministério da Agricultura, Pecuária e Abastecimento (MAPA, 2018). As variáveis avaliadas quanto às características tecnológicas da fibra foram: comprimento médio de fibra (COMP), resistência (RES), índice micronaire ou Finura (MIC ou FIN), e percentual de fibra ou rendimento de fibra ( $\mathrm{PF} \%)$. Quanto às características agronômicas foram avaliadas a produtividade de algodão em caroço (PAC), produtividade de algodão em fibra (PAF), peso médio de um capulho (P1C). Após a obtenção dos dados, estas variáveis foram inicialmente submetidas à uma análise de variância individual e em seguida à uma análise conjunta utilizando o teste $\mathrm{F}(\mathrm{P} \leq 0,01)$.

\section{RESULTADOS E DISCUSSÃO}

A condução experimental foi boa em função dos baixos CV (coeficiente de variação). Apenas Estande, Produtividade e Produtividade de Algodão em Pluma, tiveram valores acima de $10 \%$, porém dentro do esperado para essas caracteres, pois são muito influenciadas pelo ambiente. Das vinte características estudadas apenas duas (Estande e PROD) não obtiveram relevância significativas pelo teste $\mathrm{F}$, e apresentaram com altos níveis de coeficientes de variação.

Na tabela 1 encontra-se as médias das cinco cultivares avaliadas. Para a PF a BRS $416(43,6 \%)$ e a BRS 371 RF $(42,6 \%)$ se destacaram das demais. Em relação ao comprimento (UHM), a BRS 293 apresentou o maior valor $(32,5 \mathrm{~mm})$. Havendo diferença significativa para altura de plantas e produtividade.

O índice micronaire refere-se a finura de fibra. Trata-se de um caráter adimensional, largamente influenciado pela disponibilidade de celulose na parede secundária deste tecido. Esse índice estima a quantidade de fibras que irá compor a seção transversal do fio e, portanto, sua resistência e regularidade em função de comprimento. Índices > 5,0 
indicam fibras muito grossas e $<3,5$ são consideradas imaturas, ocasionando defeito nos fios e baixa afinidade do corante durante o processo de acabamento.

Tabela 1. Características Altura de Plantas (AP-cm), Produtividade (PROD-kg/ha); Produtividade de Algodão em pluma (PRODP-kg/ha); avaliadas em diferentes cultivares de algodão, em experimento conduzido sob condições de sequeiro, em Quixeramobim, Ceará

\begin{tabular}{lccc}
\hline \multirow{2}{*}{ Cultivares } & MP $(\mathrm{cm})$ & PROD $(\mathrm{kg} / \mathrm{ha})$ & $\begin{array}{c}\text { PRODP } \\
(\mathrm{kg} / \mathrm{ha})\end{array}$ \\
\cline { 2 - 4 } & $84,0 \mathrm{~b}$ & $2052,8 \mathrm{a}$ & $787,3 \mathrm{ab}$ \\
BRS 293 & $83,8 \mathrm{~b}$ & $2061,1 \mathrm{a}$ & $849,9 \mathrm{ab}$ \\
BRS 286 & $96,5 \mathrm{a}$ & $2450,0 \mathrm{a}$ & $1068,6 \mathrm{a}$ \\
BRS 416 & $100,2 \mathrm{a}$ & $2090,3 \mathrm{a}$ & $890,9 \mathrm{ab}$ \\
BRS 371 RF & $91,5 \mathrm{ab}$ & $1993,1 \mathrm{a}$ & $758,3 \mathrm{~b}$ \\
BRS Aroeira & 91,18 & 2129,44 & 870,99 \\
\hline Médias & 5,62 & 14,23 & 14,46 \\
\hline CV $(\%)$ & &
\end{tabular}

*Médias seguidas da mesma letra, na coluna, não diferem estatisticamente a $5 \%$ de probabilidade, pelo teste de Tukey. CV: Coeficiente de Variação.

De acordo com Santana, et al. (2008), os valores ideais de MIC são de 3,9 a 4,5. A estimativa do índice micronaire depende tanto da maturidade quanto da densidade linear da fibra. A maturação se dá pela deposição de celulose nas camadas secundárias, que representa $98 \%$ da espessura da parede estrutural da fibra. Das cinco cultivares estudadas apenas a BRS AROEIRA obteve um MIC aproximado ao valor ideal de 4,4 segundo Santana, et al. (2008) as demais cultivares ficaram entre 4,6 e 5,2 na conclusão temos fibras grossas e para a indústria têxtil tecnicamente não é recomendado pois o fio não apresenta um valor de resistência. A relevância dessa característica é que CSP é um indicador da resistência dos fios, que depende essencialmente das fibras individuais. De acordo com as exigências da indústria têxtil, os valores ideais para índice de fiabilidade vão de 2000 a 2500 (FUNDAÇÃO MT, 1999). Entre os valores de fiabilidade obtivemos resultados que variam de 2704,2 a 3185,6 acima das exigências da indústria têxtil.

\section{CONCLUSÃO}

Há variabilidade fenotípica para a fibra de cultivares de algodoeiro sob condições de sequeiro

As cultivares BRS 416, BRS 286 e BRS 293 são as de melhores desempenhos para produtividade de pluma e para as características de fibras.

\section{REFERÊNCIAS}

ABRAPA, Associação Brasileira dos Produtores de Algodão (2018).

Disponível em:<www.abrapa.com.br/paginas/dados/produção-mundialalgodão_backup2017-.aspx>. Acesso em: 10 dez. 2018.

AQUINO, L. A.; AQUINO, R. F. B. A.; SILVA, T. C.; SANTOS, D. F.; BERGER, P. G. Aplicação do fósforo e da irrigação na absorção e exportação de nutrientes pelo algodoeiro. Revista Brasileira de Engenharia Agrícola e Ambiental, v. 16, n. 4, p. 355-361, 2012.
BALOCH, M. J.; KHAN, N. U.; RAJPUT, M. A.; JATOI, W. A.; GUL, S.; RIND, I. H.; VEESAR, N. F. Yield related morphological measures of short duration cotton genotypes. Journal of Animal and Plant Sciences, v. 24, n. 4, p. 1198$1211,2014$.

BEDNARZ, C. W.; NICHOLS, R. L. Phenological and morphological components of cotton crop maturity. Crop science, v. 45, n. 4, p. 1497-1503, 2005.

CARVALHO, L. P.; ANDRADE, F. P.; SILVA FILHO, J. L. Cultivares de algodão colorido no Brasil. Revista Brasileira de Oleaginosas e Fibrosas, v. 15, n. 1, p. 37- 44, 2011.

CARVALHO, L. P.; FARIAS, F. J. C.; RODRIGUES, J. I. S. Selection for increased fiber length in cotton progenies from Acala and non-Acala types. Crop Science, v. 55, p. 1-7, 2015.

CONAB, Companhia Nacional de Abastecimento. Algodão: análise mensal. Março de 2018. Disponível em: <conab.gov.br>. Acesso em: $17 \mathrm{dez} 2018$.

DHIVYA, R.; AMALABALU, P.; PUSHPA, R.; KAVITHAMANI, D. Variability, heritability and genetic advance in upland cotton (Gossypium hirsutum L.). African Journal of Plant Science, v. 8, n. 1, p. 1-5, 2014.

EMBRAPA, Empresa Brasileira de Pesquisa Agropecuária. Embrapa, IMAmt e Abrapa firmam parceria para desenvolver algodão resistente ao bicudo. 2017. Disponível em: <embrapa.br/busca-de-noticias/-/noticia/26418363/embrapaimamt-e-abrapa-firmam-parceria-para-desenvolver-algodaoresistente-ao-bicudo>. Acesso em: 06 dez. 2018.

FARIAS, F. J. C.; SMITH, C. W.; MORELLO, C. L.; HAGUE, S.; NG, E. H.; JOY,K. S.; FARIAS, F. C. Genetic mean analysis of earliness among brazilian and U.S. upland cotton. Proceedings of Beltwide Cotton Conference. Orlando: Florida. 44p. 2012.

FREIRE, J. C.; MORELLO, C. L.; FARIAS, F. J. C.; FILHO, VITAL NETO, F. C.; PEDROSA, M. B.; SUINAGA, F. A.; COSTA, J. N.; ANDRADE, F. P. Objetivo e métodos usados nos programas de melhoramento do algodão. In: BELTRÃO, N. E. M.; AZEVEDO, D. M. P. (Orgs.). O agronegócio do algodão no Brasil. 2. ed. Brasília: Embrapa Informação Tecnológica, 2008. p. 299-323.

FREIRE, E. C. Melhoramento no Brasil. In: Algodão: do plantio à colheita. BORÉM, A.; FREIRE, E. C. (Eds.). Viçosa: Editora UFV, 2014. p. 113-132.

FUNDAÇÃO MT (Rondonópolis, MT). Liderança e Competividade. Rondonópolis, MT, p.182, 1999. Acessado em 17 dez 2018

GWATHMEY, C. O.; BANGE, M. P.; BRODRICK, R. Cotton crop maturity: A compendium of measures and predictors. Field Crops Research, v. 191, p. 41-53, 2016.

HEILMAN, M.D.; NAMKEN, L.N.; SUMMY, K.R. Sistema deprodução de algodões de ciclo curto para áreas infestadas pelo bicudo. In: BARBOSA, S.; LUKEFHAR, M.J.; BRAGA 
SOBRINHO, R.(Ed.). O bicudo do algodoeiro. Brasília: Embrapa-DDT, 1986. p. 253-274.

PEDROSA, M. B; FREIRE, E. C; COSTA, J. N; ANDRADE, F. P. Estimativa das capacidades combinatórias em híbridos $\mathrm{F}_{1 \mathrm{~s}}$ de algodoeiro (Gossypium hirsutum L.) irrigado no Estado do Rio Grande do Norte. Revista de Oleaginosas e Fibrosas. v.5, n. 3, p. 439-445, 2001.

PENNA, J. C. V. Melhoramento do algodão. In: BORÉM, A. (Ed). Melhoramento de espécies cultivadas. Viçosa: Editora da UFV, p. 15-53, 2005.

PETTIGREW, W. T. Moisture deficit effects on cotton lint yield, yield components, and boll distribution. Agronomy Journal, v. 96, n. 2, p. 377-383, 2004.

ROACH, S. H.; CULP, T. W. An evaluation of three early maturing cotton cultivars for production potential and insect damage in reduced- and conventional-tillage systems. Journal of Agricultural Entomology, v. 1, n. 3, p. 249-255, 1984.

SANTANA, J. C. F.; WANDERLEY, M. J. R.; BELTRÃO, N. E. M.; AZEVEDO, D. M. P.; LEÃO, A. B.; VIEIRA, D. J. Características da fibra e do fio do algodão: análise e interpretação dos resultados. In: BELTRÃO N. E. M. AZEVEDO D. M. P. O agronegócio do algodão no Brasil. Brasília: Embrapa Informação Tecnológica v.2, p.1099-1120, 2008.

SOUZA, J. G.; BELTRÃO, N. E. M.; AZEVEDO, D. M. P.; ALBUQUERQUE, W. G.; LIMA, R. L. S.; CARDOSO, G. D. Fisiologia. In: BELTRÃO, N. E. M.;ULLAH, A.; SUN, H.; YANG, X.; ZHANG, X. Drought coping strategies in cotton: increaded crop per drop. Plant Biotechnology Journal, v. 15, n. 3, p. 271-284, 2017.

STEWART, J. M. Fiber initiation on the cotton ovule (Gossypium hirsutum). American Journal of Botany, v. 62, n. 7, p. 723-730, 1975.

VIDAL NETO, F. C.; FREIRE, E. C. Melhoramento genético do algodão. In: VIDAL NETO, F. C.; CAVALCANTI, J. J. V. (Eds.). Melhoramento genético de plantas no Nordeste. Brasília: Embrapa, 2013. p. 49-83.

VIDAL NETO, F. das C.; FREIRE, E. C. Melhoramento genético do algodoeiro. In:VIDAL NETO, $F$ das $C$.; CAVALCANTI, J. J. V. (Ed.) Melhoramento genético de plantas no Nordeste.1 ed. Brasilia, DF: Embrapa, v. 1,2013.p.49-84

SDA, United States Department of Agriculture. Cotton: world markets and trade. Abril de 2018. Disponível em: <fas.usda.gov>. Acesso em: 18 dez. 2018.

ZONTA, J. H.; BEZERRA, J. R. C.; SOFIATTI, V.; FARIAS, F. J. C.;CARVALHO, L. P. de. Efeito da irrigação no rendimento e qualidade de fibra em cultivares de algodão herbáceo. ', v. 28, n. 4, p. 43-52, 2015. 